\title{
CORRIGENDUM
}

\section{Coherent coupling between distant excitons revealed by two-dimensional nonlinear}

hyperspectral imaging

J. Kasprzak, B. Patton, V. Savona \& W. Langbein

Nature Photon. 5, 57-63 (2011); published online 19 December 2010; corrected after print 7 January 2011.

The left-hand dash-dot line shown in Fig. 2b was incorrectly labelled as "X states", when it should have read "XX states".

This error has been corrected in the HTML and PDF versions of the Article. 